\title{
Systematic review and meta-analysis of laparoscopic and open gastrectomy for advanced gastric cancer
}

\author{
Ke Chen, Xiao-Wu Xu, Yi-Ping Mou*, Yu Pan, Yu-Cheng Zhou, Ren-Chao Zhang and Di Wu
}

\begin{abstract}
Background: The use of laparoscopic gastrectomy (LG) in advanced gastric cancer (AGC) remains a controversial topic, mainly because of doubts about its oncologic validity. This study is a systematic review and meta-analysis of the available evidence.

Methods: A comprehensive search was performed until June 2013 to identify comparative studies evaluating survival rates, recurrence rates, surgical outcomes and complications. Pooled risk ratios (RR) and weighted mean differences (WMD) with 95\% confidence intervals (Cl) were calculated using the random effects model. Data synthesis and statistical analysis were carried out using RevMan 5.1 software.

Results: Fifteen trials were involved in this analysis. Compared to open gastrectomy (OG), LG involved a longer operating time $(\mathrm{WMD}=48.67 \mathrm{~min}, 95 \% \mathrm{Cl} 34.09$ to 63.26, $P<0.001)$; less blood loss (WMD $=-139.01 \mathrm{ml}, 95 \%$ $\mathrm{Cl}-174.57$ to $-103.44, P<0.001$ ); earlier time to flatus (WMD $=-0.79$ days, $95 \% \mathrm{Cl}-1.14$ to $-0.44, P<0.001$ ); shorter hospital stay (WMD $=-3.11$ days, $95 \% \mathrm{Cl}-4.13$ to $-2.09, P<0.001)$; and a decrease in complications ( $R R=0.74,95 \% \mathrm{Cl} 0.61$ to $0.90, P=0.003$ ). There was no significant difference in the number of harvested lymph nodes, margin distance, mortality, cancer recurrence rate and long-term survival rate between the AGC patients treated with LG or OG $(P>0.05)$.

Conclusions: Despite a longer operation, LG is a safe technical alternative to OG for AGC with a lower complication rate and enhanced postoperative recovery. Moreover, there were similar outcomes between both approaches in terms of cancer recurrence and the long-term survival rate. Because of the limitation of this study, methodologically high-quality studies are needed for further evaluation.
\end{abstract}

\section{Background}

Although the annual incidence of and mortality from gastric cancer have been decreasing yearly worldwide, gastric cancer still accounts for more than $10 \%$ of cancer deaths worldwide and is the second most frequent cause of cancer death after lung cancer [1,2]. Adjuvant chemotherapy improves the survival of these patients [3,4], but radical gastrectomy with regional lymph node dissection still remains the only potentially curative treatment available for gastric adenocarcinoma [5,6].

Since the first report of laparoscopic gastrectomy (LG) for early gastric cancer (EGC) by Kitano [7], it has

\footnotetext{
*Correspondence: mouyiping2002@163.com

Department of General Surgery, Sir Run Run Shaw Hospital, School of Medicine, Zhejiang University, 3 East Qingchun Road, Hangzhou, Zhejiang Province 310016, China
}

undergone rapid development and gained popularity in the past 20 years. Compared to traditional open gastrectomy (OG), most studies have reported that LG can achieve better cosmesis, shorter hospital stay, faster recovery and better postoperative quality of life [8-13]. However, most of these studies focus on EGC. LG for advanced gastric cancer (AGC) remains controversial and has not achieved universal acceptance because of its uncertain oncological safety, particularly given the technical difficulty of lymphadenectomy for metastatic lymph nodes [14]. Meanwhile, there have been few long-term follow-up results regarding the oncological adequacy of laparoscopic surgery compared to that of open surgery for AGC.

Although several meta-analyses and systematic reviews have demonstrated the safety and oncological effect of LG for EGC [15-19], such studies have not been

\section{Biomed Central}


conducted for the potential benefits and disadvantages of LG for AGC. The aim of this study was to compare LG with OG with respect to morbidity, mortality, intraoperative outcomes and functional recovery. Longterm outcomes after LG and OG in patients with AGC were evaluated in a systematic review of the literature, and meta-analyses were performed.

\section{Methods}

\section{Literature search}

A systematic search using the following keywords, "laparoscopy", "laparoscopic", "gastric cancer", "gastric carcinoma" and "gastrectomy", was performed through the following bibliographic databases, PubMed, Web of Science and Cochrane Library, for literature comparing LG and OG published between January 1995 and June 2013, and we broadened the search range by browsing the related summary, methods and references of retrieved articles. The language of the publications was confined to English. Two investigators reviewed the titles and abstracts, and assessed the full text to establish eligibility.

\section{Study selection criteria}

All clinical studies needed to meet the following criteria for the meta-analysis: (1) being published in English with data comparing LG and OG for AGC; (2) having clear case selection criteria and surgical methods; they had to contain long-term outcomes such as tumor recurrence and survival rate; (3) articles referring only or predominantly to AGC, because it is difficult to confirm AGC preoperatively [20]. However, articles with significant differences in tumor stages between groups were excluded. (4) If there was an overlap between authors or centers, only the higher quality or more recent literature was selected. However, articles from the same authors or centers but with different patient cohorts were included.

\section{Data extraction and quality assessment}

Two authors independently extracted the data using a unified data sheet and decided upon the controversial issues through discussion. Extracted data included the author, study period, geographical region, number of patients, operating time, blood loss, number of retrieved lymph nodes, proximal and distal margin distance, time to flatus, time to oral intake, length of hospital stay, morbidity and mortality, tumor recurrence and survival rate. Postoperative complications were classified as medical (cardiovascular, respiratory or metabolic events; nonsurgical infections; deep venous thrombosis; pulmonary embolism) or surgical (any anastomotic leakage or fistula, any complication that required reoperation, intraabdominal collections, wound complications, bleeding events, pancreatitis, ileus, delayed gastric emptying and anastomotic stricture). This classification system is based on the Memorial Sloan-Kettering Cancer Center complication reporting system [21]. If necessary, the first authors were contacted to retrieve further information.

Randomized controlled trials (RCTs) were evaluated by the Jadad composite scale. High-quality trials scored more than 2 out of a maximum possible score of 5 . The Newcastle-Ottawa Quality Assessment Scale (NOS) was used for quality assessment of observational studies. A threshold of six stars or above has been considered indicative of high quality.

\section{Statistical analysis}

The meta-analysis was performed in line with recommendations from the Cochrane Collaboration and the Quality of Reporting of Meta-Analyses guidelines [22,23]. Continuous variables were assessed using the weighted mean difference (WMD), and dichotomous variables were analyzed using the risk ratio (RR). If the study provided medians and ranges instead of means and standard deviations (SDs), we estimated the means and SDs as described by Hozo et al. [24]. To account for clinical heterogeneity, which refers to diversity in a sense that is relevant for clinical situations, we used the random effects model based on DerSimonian and Laird's method. Potential publication bias was determined by conducting informal visual inspection of funnel plots based on the complications. Data analyses were performed using Review Manage version 5.1 (RevMan 5.1) software downloaded from the Cochrane Library. $P<0.05$ was considered statistically significant.

\section{Results}

\section{Studies selected}

The initial search strategy retrieved 2,068 publications in English. After the titles and abstracts had been reviewed, papers without comparison of LG and OG for AGC were excluded, which left 21 comparative studies, 6 [25-30] of which did not meet the inclusion criteria and were excluded. This left a total of two RCTs and $13 \mathrm{ob-}$ servational studies [31-45], all of which were accessible in full-text format. A flow chart of the search strategies is illustrated in Figure 1.

\section{Study characteristics and quality}

A total of 2,519 patients were included in the analysis with 1,327 undergoing LG (52.7\%) and 1,192 undergoing OG (47.3\%). They represent international experience including data from five different countries (six from China, four from Korea, three from Italy, one from Japan and one from Chile). Two RCTs got Jadad scores of 2 and 3, respectively. According to the NOS, 2 out of the 13 observational studies got 7 stars, 6 articles got 8 stars, and the remaining 5 got 9 stars. The characteristics and 


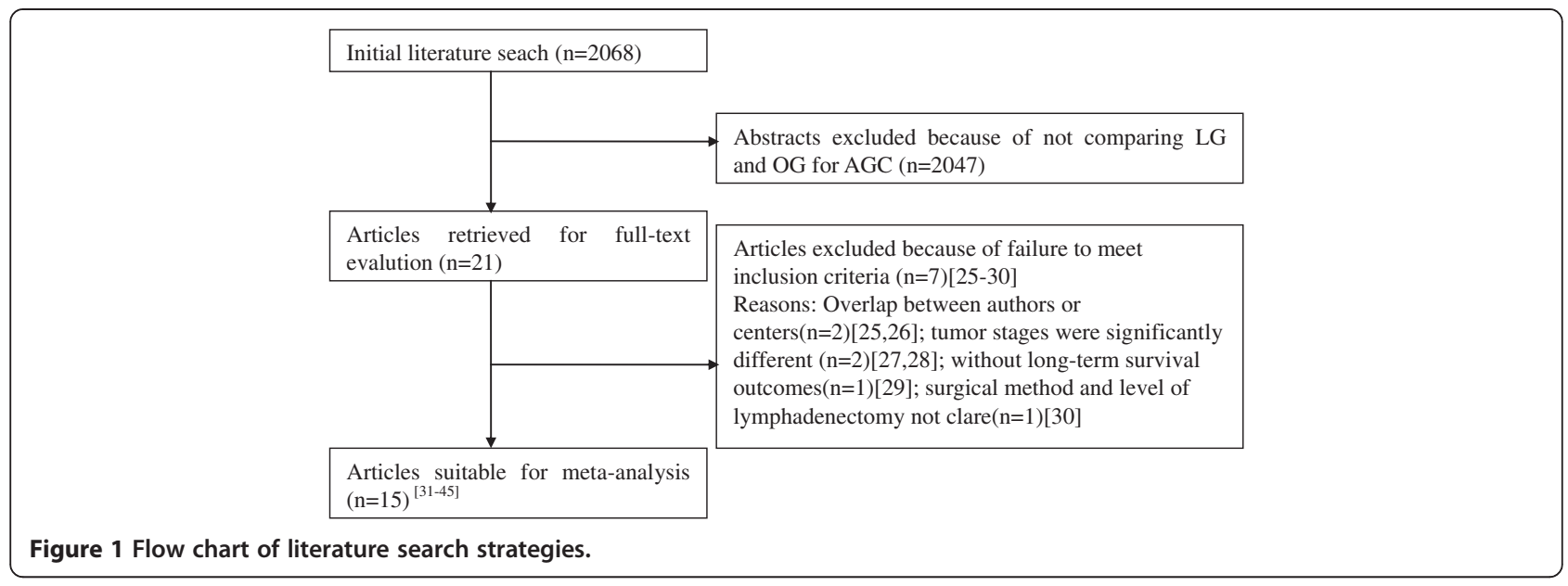

methodological quality assessment scores of the included studies are shown in Table 1.

\section{Intraoperative effects}

The mean operating time of LG was 48.67 min longer than for OG (WMD = $48.67 \mathrm{~min}, 95 \%$ CI 34.09 to 63.26 , $P<0.001)$. The intraoperative blood loss was lower in LG than in OG (WMD $=-139.01 \mathrm{ml}, 95 \% \mathrm{CI}-174.57$ to $-103.44, P<0.001$ ). All studies contained the number of retrieved lymph nodes. The difference in the mean number of retrieved lymph nodes between LG and OG was not significant in the pooled data (WMD $=-0.07$, $95 \% \mathrm{CI}-1.03$ to $0.89, P=0.88$ ) (Figure 2 ). Meta-analysis of the distal margin distance showed no significant difference between the two groups (WMD $=0.08 \mathrm{~cm}, 95 \%$ CI -0.16 to $0.32, P=0.50$ ). However, the proximal margin distance of OG was longer than that of LG with a marginal difference $(\mathrm{WMD}=-0.26 \mathrm{~cm}, 95 \% \mathrm{CI}-0.54$ to $0.01, P=0.06$ ). All intraoperative effect outcomes are summarized in Table 2.

\section{Postoperative outcome}

The mean time to first flatus was shorter in LG than in OG $(\mathrm{WMD}=-0.79 \mathrm{~d}, 95 \% \mathrm{CI}-1.14$ to $-0.44, P<$ $0.001)$, as was the time to restart oral intake after surgery (WMD $=-1.06 \mathrm{~d}, 95 \% \mathrm{CI}-1.63$ to $-0.50, P<0.001$ ). A shorter hospital stay was also observed in the LG group (WMD $=-3.11 \mathrm{~d}, 95 \% \mathrm{CI}-4.13$ to $-2.09, P<0.001)$.

Mortality was described in seven studies, and there was no significant difference in postoperative mortality $(\mathrm{RR}=$ $0.78,95 \%$ CI 0.30 to $2.04, P=0.61$ ). The rate of overall postoperative complications was lower for $\mathrm{LG}(\mathrm{RR}=0.74$, 95\% CI 0.61 to $0.90, P=0.003$ ) (Figure 3). Visual inspection of the funnel plot revealed symmetry, indicating no serious publication bias (Figure 4). After further analysis, surgical complications were also lower for $L G(R R=0.73$, $95 \%$ CI 0.58 to $0.92, P=0.007)$. In analyzing the specific complications, wound infection and ileus were lower for
LG (wound infection: $\mathrm{RR}=0.53,95 \% \mathrm{CI} 0.33$ to $0.85, P=$ 0.009; ileus: $\mathrm{RR}=0.27,95 \% \mathrm{CI} 0.09$ to $0.75, P=0.01$ ). Other surgical complications such as anastomotic leakage, intra-abdominal collections, bleeding or anastomotic stricture were similar between groups $(P>0.05)$. Besides, LG was associated with a marginal reduction in medical complications ( $\mathrm{RR}=0.65,95 \% \mathrm{CI} 0.41$ to $1.02, P=0.06$ ) with a possible contribution from respiratory complications $(\mathrm{RR}=0.57,95 \% \mathrm{CI} 0.30$ to $1.10, P=0.09)$. All postoperative outcomes are summarized in Table 2.

\section{Recurrence and long-term survival rate}

Ten studies reported the cancer recurrence [31-35,39, 41-43,45]. The recurrence risk in LG was 29.9\% (288/ 964) and 30.5\% (288/943) in OG, but the difference between LG and OG was not significant ( $R R=0.94,95 \%$ CI 0.83 to $1.08, P=0.38$ ) (Figure 5). The available data about recurrence patterns and specific recurrent sites are summarized in Table 3.

Another four studies [31,43-45] available reported no port-site metastases in the LG group. Hwang et al. [34] reported a port-site recurrence 10 months after LG. Zhao et al. [39] reported a case of port-site recurrence 13 months after LG group; a case of incision metastasis and a case of metastasis in the orifice of the abdominal drain tube 27 and 9 months, respectively, after OG group. Moison et al. [43] reported tumors recurred in distant sites in three patients in the LG and in two patients in the OG group, and a recurrence in the remnant stomach in the LG group. Shinohara et al. [45] reported 53 recurrences in the LG group: 29 (54.7\%) from peritoneal recurrence, 23 (43.4\%) from distant or hematogenous recurrence and 15 (28.3\%) from locoregional or lymphatic recurrence; the corresponding findings in the OG group were 17 (50\%), 15 (44.1\%) and 11 (32.6\%), respectively.

Twelve studies reported postoperative survival rates [31-33,36,37,39-45], all of which did not find significant differences in survival rates between groups. Although 
Table 1 Characteristics of included studies

\begin{tabular}{|c|c|c|c|c|c|c|c|c|c|c|c|c|}
\hline \multirow[t]{2}{*}{ Author } & \multirow[t]{2}{*}{ Country } & \multirow[t]{2}{*}{ Study type } & \multirow{2}{*}{$\begin{array}{l}\text { Publication } \\
\text { year }\end{array}$} & \multirow{2}{*}{$\begin{array}{l}\text { Study } \\
\text { period }\end{array}$} & \multicolumn{2}{|c|}{ Sample size } & \multirow{2}{*}{$\begin{array}{c}\text { Level of } \\
\text { lymphadenectomy }\end{array}$} & \multirow{2}{*}{$\begin{array}{c}\text { Surgical } \\
\text { extension }\end{array}$} & \multirow[t]{2}{*}{ Reconstruction } & \multicolumn{2}{|c|}{ Adjuvant chemotherapy (\%) } & \multirow{2}{*}{$\begin{array}{l}\text { Quality } \\
\text { scores }\end{array}$} \\
\hline & & & & & LG & $\overline{\text { OG }}$ & & & & LG & OG & \\
\hline Huscher & Italy & RCT & 2005 & $1992-1996$ & 30 & 29 & D1, D2 & DG & B-II, R-Y & NR & NR & $3^{*}$ \\
\hline Hur & Korea & Retrospective & 2008 & $2004-2007$ & 26 & 25 & D2 & DG & $B-I, B-I I$ & 76.9 & 68 & 8 \\
\hline $\mathrm{Du} \times \mathrm{H}$ & China & Retrospective & 2009 & 2004-2008 & 78 & 90 & D2 & DG & $B-I, B-I I$ & NR & NR & 7 \\
\hline Hwang & Korea & Retrospective & 2009 & $2004-2007$ & 45 & 83 & $\mathrm{D} 1+\alpha / \beta, \mathrm{D} 2$ & DG & $B-\mid, B-I I$ & 93.2 & 89.0 & 7 \\
\hline Du J & China & Retrospective & 2010 & 2005-2009 & 82 & 94 & D2 & TG & $R-Y$ & 100 & 100 & 8 \\
\hline Cai & China & RCT & 2011 & 2008-2009 & 49 & 47 & D2 & $\mathrm{DG}, \mathrm{PG}, \mathrm{TG}$ & $B-I, B-I I, R-Y$ & 100 & 100 & $2^{*}$ \\
\hline Scatizzi & Italy & Retrospective & 2011 & 2006-2009 & 30 & 30 & D2 & DG & $R-Y$ & NR & $N R$ & 8 \\
\hline Shuang & China & Retrospective & 2011 & $2005-2007$ & 35 & 35 & D2 & DG & $B-\|$ & NR & $N R$ & 8 \\
\hline Zhao & China & Retrospective & 2011 & $2004-2009$ & 346 & 313 & $\mathrm{D} 1+\alpha / \beta, \mathrm{D} 2$ & DG & B-I, B-II & 93.1 & 91.7 & 9 \\
\hline Chen & China & Retrospective & 2012 & $2008-2010$ & 224 & 112 & D2 & $\mathrm{DG}, \mathrm{TG}$ & B-I, B-II, R-Y & $N R$ & NR & 8 \\
\hline Chun & Korea & Retrospective & 2012 & 2004-2009 & 52 & 67 & D2 & DG & $B-I, B-I I, R-Y$ & NR & NR & 9 \\
\hline Kim & Korea & Retrospective & 2012 & 1999-2007 & 88 & 88 & D2 & $\mathrm{DG}, \mathrm{TG}$ & B-I, B-II, R-Y & NR & NR & 9 \\
\hline Moisan & Chile & Retrospective & 2012 & $2005-2010$ & 31 & 31 & $D 1+\alpha / \beta, D 2$ & $\mathrm{DG}, \mathrm{TG}$ & $B-\|, R-Y$ & 19.4 & 22.6 & 8 \\
\hline Siani & Italy & Retrospective & 2012 & 2003-2009 & 25 & 25 & $D 1+\alpha / \beta, D 2$ & TG & $R-Y$ & NR & NR & 9 \\
\hline Shinohara & Japan & Retrospective & 2013 & $1998-2008$ & 186 & 123 & D2 & $\mathrm{DG}, \mathrm{PG}, \mathrm{TG}$ & $B-I, R-Y$ & 61.3 & 58.5 & 9 \\
\hline
\end{tabular}

Abbreviations: RCT Randomized controlled trial, LG Laparoscopic gastrectomy, OG Open gastrectomy, DG Distal gastrectomy, PG Proximal gastrectomy, TG Total gastrectomy, $B-I$ Billroth-I, $B-I /$ Billroth-II, $R-Y$ Roux-en-Y, NR Not reported. *Jadad scores. 


\begin{tabular}{|c|c|c|c|c|c|c|c|c|c|}
\hline Study or Subgroup & Mean & $\begin{array}{l}\text { LG } \\
\text { SD }\end{array}$ & Total & Mean & $\begin{array}{l}\mathrm{OG} \\
\mathrm{SD}\end{array}$ & Total & Weight & $\begin{array}{l}\text { Mean Difference } \\
\text { IV. Random, } 95 \% \mathrm{Cl} \text { Year }\end{array}$ & $\begin{array}{c}\text { Mean Difference } \\
\text { IV. Random, } 95 \% \mathrm{Cl}\end{array}$ \\
\hline Huscher & 30 & 14.9 & 30 & 33.4 & 17.4 & 29 & $1.3 \%$ & $-3.40[-11.68,4.88] 2005$ & \\
\hline Hur & 30.5 & 16.5 & 26 & 35 & 17.8 & 25 & $1.0 \%$ & $-4.50[-13.93,4.93] 2008$ & \\
\hline Hwang & 35.6 & 14.2 & 45 & 38.3 & 11.4 & 83 & $3.6 \%$ & $-2.70[-7.52,2.12] 2009$ & - \\
\hline Du XH & 23.5 & 6 & 78 & 21 & 7.5 & 90 & $14.7 \%$ & $2.50[0.46,4.54] 2009$ & - \\
\hline Du J & 34.2 & 13.5 & 82 & 36.4 & 19.1 & 94 & $3.6 \%$ & $-2.20[-7.04,2.64] 2010$ & \\
\hline Scatizzi & 31 & 11 & 30 & 37 & 20.3 & 30 & $1.3 \%$ & $-6.00[-14.26,2.26] 2011$ & - \\
\hline Cai & 23 & 2.7 & 49 & 22.9 & 2.4 & 47 & $29.5 \%$ & $0.10[-0.92,1.12] 2011$ & - \\
\hline Shuang & 35 & 14 & 35 & 38 & 15 & 35 & $1.9 \%$ & $-3.00[-9.80,3.80] 2011$ & \\
\hline Zhao & 33.2 & 12.5 & 346 & 32.8 & 15.6 & 313 & $13.5 \%$ & $0.40[-1.77,2.57] 2011$ & - \\
\hline Chun & 39.1 & 15.2 & 52 & 39.3 & 11.2 & 67 & $3.5 \%$ & $-0.20[-5.13,4.73] 2012$ & \\
\hline Moisan & 35 & 14.8 & 31 & 39 & 24.3 & 31 & $0.9 \%$ & $-4.00[-14.02,6.02] 2012$ & \\
\hline Siani & 35 & 18 & 25 & 40 & 16 & 25 & $1.0 \%$ & $-5.00[-14.44,4.44] 2012$ & \\
\hline Chen & 30.6 & 10.1 & 224 & 30.3 & 8.6 & 112 & $14.5 \%$ & $0.30[-1.77,2.37] 2012$ & - \\
\hline Kim & 38.3 & 14.3 & 88 & 41.8 & 15.3 & 88 & $4.3 \%$ & $-3.50[-7.88,0.88] 2012$ & \\
\hline Shinohara & 45.3 & 16.9 & 186 & 43.8 & 17.2 & 123 & $5.3 \%$ & $1.50[-2.39,5.39] 2013$ & \\
\hline Total $(95 \% \mathrm{Cl})$ & & & 1327 & & & 1192 & $100.0 \%$ & $-0.07[-1.03,0.89]$ & \\
\hline \multicolumn{9}{|c|}{$\begin{array}{l}\text { Heterogeneity: } \mathrm{Tau}^{2}=0.54 ; \mathrm{Chi}^{2}=16.94 \text {, df } \\
\text { Test for overall effect: } Z=0.15(P=0.88)\end{array}$} & $\begin{array}{ccccc}-10 & -5 & 0 & 5 & 10 \\
\text { Favours OG } & \text { Favours LG }\end{array}$ \\
\hline
\end{tabular}

Shuang et al. [38] did not report specific survival rates, they also found no significant difference in the survival rates between the two groups after 50 months of followup $(P>0.05)$. Meta-analysis of these available data demonstrated that the disease-free survival (DFS) rate was not significantly different in participants who received LG compared with OG (3-year: RR $=1.11,95 \%$ CI 0.75 to $1.65, P=0.59$; 5 -year: $\mathrm{RR}=1.03,95 \%$ CI 0.93 to 1.14 , $P=0.56$ ) (Figure 6), nor was the overall survival (OS) rate (1-year: $\mathrm{RR}=1.01,95 \% \mathrm{CI} 0.96$ to $1.05, P=0.79$; 3 year: $\mathrm{RR}=1.08,95 \% \mathrm{CI} 0.99$ to $1.17, P=0.07$; 5 -year: $\mathrm{RR}=1.03,95 \%$ CI 0.96 to $1.11, P=0.39$ ) (Figure 6). The systematic review outcomes of long-term survival rates are summarized in Table 4.

\section{Discussion}

RCTs are the most ideal tool for meta-analysis. However, it is difficult to conduct a high-quality RCT to evaluate a new surgical intervention because of obstacles such as learning curve effects, ethical and cultural resistance, and urgent or unexpected conditions during the operation. For these reasons, to include non-RCTs is an appropriate strategy to extend the source of evidence. Therefore, our meta-analysis synthesized the existing observational studies

Table 2 Pooled short-term outcomes of meta-analysis

\begin{tabular}{|c|c|c|c|c|c|c|c|}
\hline \multirow[t]{2}{*}{ Outcomes } & \multirow{2}{*}{$\begin{array}{l}\text { No. of } \\
\text { studies }\end{array}$} & \multicolumn{2}{|c|}{ Sample size } & \multirow{2}{*}{$\begin{array}{l}\text { Heterogeneity } \\
\qquad\left(P, I^{2}\right)\end{array}$} & \multirow{2}{*}{$\begin{array}{l}\text { Overall effect } \\
\text { size }\end{array}$} & \multirow{2}{*}{$\begin{array}{c}95 \% \mathrm{Cl} \text { of } \\
\text { overall effect }\end{array}$} & \multirow[t]{2}{*}{$P$ value } \\
\hline & & LG & OG & & & & \\
\hline Operating time (min) & 15 & 1327 & 1192 & $<0.001,93 \%$ & WMD = 48.67 & $34.09-63.06$ & $<0.001$ \\
\hline Blood loss (ml) & 12 & 1157 & 1007 & $<0.001,91 \%$ & WMD $=-139.01$ & $-174.57--103.44$ & $<0.001$ \\
\hline Retrieved lymph nodes & 15 & 1327 & 1192 & $0.26,17 \%$ & WMD $=-0.07$ & $-1.03-0.89$ & 0.88 \\
\hline Proximal margin (cm) & 6 & 588 & 607 & $0.27,21 \%$ & WMD $=-0.26$ & $-0.54-0.01$ & 0.06 \\
\hline Distal margin $(\mathrm{cm})$ & 4 & 517 & 499 & $0.86,0 \%$ & WMD $=0.08$ & $-0.16-0.32$ & 0.50 \\
\hline Analgesics given (days) & 4 & 192 & 242 & $<0.001,89 \%$ & WMD $=-1.57$ & $-2.40--0.74$ & $<0.001$ \\
\hline Time to ambulation (days) & 6 & 913 & 755 & $<0.001,96 \%$ & WMD $=-1.01$ & $-1.56--0.45$ & $<0.001$ \\
\hline Time to first flatus (days) & 11 & 1045 & 974 & $<0.001,94 \%$ & WMD $=-0.79$ & $-1.14--0.44$ & $<0.001$ \\
\hline Time to oral intake (days) & 9 & 967 & 793 & $<0.001,87 \%$ & WMD $=-1.06$ & $-1.63--0.50$ & $<0.001$ \\
\hline Hospital stay (days) & 14 & 1238 & 1091 & $<0.001,84 \%$ & WMD $=-3.11$ & $-4.13--2.09$ & $<0.001$ \\
\hline Overall complications & 15 & 1327 & 1192 & $0.66,0 \%$ & $\mathrm{RR}=0.74$ & $0.61-0.90$ & 0.003 \\
\hline Surgical complications & 15 & 1,327 & 1,192 & $0.56,0 \%$ & $\mathrm{RR}=0.73$ & $0.58-0.92$ & 0.007 \\
\hline Medical complications & 12 & 868 & 754 & $0.40,5 \%$ & $\mathrm{RR}=0.65$ & $0.41-1.02$ & 0.06 \\
\hline Mortality & 7 & 965 & 821 & $0.75,0 \%$ & $\mathrm{RR}=0.78$ & $0.30-2.04$ & 0.61 \\
\hline
\end{tabular}

Abbreviations: WMD Weighted mean difference, RR Risk ratio. 


\begin{tabular}{|c|c|c|c|c|c|c|c|c|c|c|}
\hline Study or Subgroup & $\begin{array}{r}\text { LG } \\
\text { Events }\end{array}$ & Total & $\begin{array}{r}O G \\
\text { Events }\end{array}$ & Total & Weight & $\begin{array}{c}\text { Risk Ratio } \\
\text { M-H, Random, } 95 \% \mathrm{Cl}\end{array}$ & Year & & $\begin{array}{r}\text { Risk } \\
\text { M-H, Rand } \\
\end{array}$ & $\begin{array}{l}\text { Ratio } \\
\text { lom. } 95 \% \mathrm{Cl}\end{array}$ \\
\hline Huscher & 7 & 30 & 8 & 29 & $5.0 \%$ & $0.85[0.35,2.03]$ & 2005 & & & \\
\hline Hur & 4 & 26 & 4 & 25 & $2.4 \%$ & $0.96[0.27,3.43]$ & 2008 & & & \\
\hline Du XH & 6 & 78 & 10 & 90 & $4.1 \%$ & $0.69[0.26,1.82]$ & 2009 & & & \\
\hline Hwang & 7 & 45 & 10 & 83 & $4.8 \%$ & $1.29[0.53,3.16]$ & 2009 & & & \\
\hline $\mathrm{Du} J$ & 8 & 82 & 23 & 94 & $6.9 \%$ & $0.40[0.19,0.84]$ & 2010 & & & \\
\hline Zhao & 24 & 346 & 41 & 313 & $16.7 \%$ & $0.53[0.33,0.86]$ & 2011 & & & \\
\hline Shuang & 2 & 35 & 3 & 35 & $1.3 \%$ & $0.67[0.12,3.75]$ & 2011 & & & \\
\hline Scatizzi & 2 & 30 & 8 & 30 & $1.8 \%$ & $0.25[0.06,1.08]$ & 2011 & & & \\
\hline Cai & 6 & 49 & 9 & 47 & $4.3 \%$ & $0.64[0.25,1.66]$ & 2011 & & & \\
\hline Siani & 4 & 25 & 5 & 25 & $2.7 \%$ & $0.80[0.24,2.64]$ & 2012 & & & \\
\hline Moisan & 8 & 31 & 6 & 31 & $4.4 \%$ & $1.33[0.52,3.39]$ & 2012 & & & \\
\hline Kim & 7 & 88 & 7 & 88 & $3.8 \%$ & $1.00[0.37,2.73]$ & 2012 & & & \\
\hline Chun & 5 & 52 & 6 & 67 & $3.0 \%$ & $1.07[0.35,3.32]$ & 2012 & & & \\
\hline Chen & 25 & 224 & 17 & 112 & $11.8 \%$ & $0.74[0.41,1.30]$ & 2012 & & & . \\
\hline Shinohara & 45 & 186 & 35 & 123 & $26.9 \%$ & $0.85[0.58,1.24]$ & 2013 & & . & - \\
\hline Total $(95 \% \mathrm{Cl})$ & & 1327 & & 1192 & $100.0 \%$ & $0.74[0.61,0.90]$ & & & & \\
\hline Total events & 160 & & 192 & & & & & & & \\
\hline \multicolumn{8}{|c|}{ Heterogeneity: $\mathrm{Tau}^{2}=0.00 ; \mathrm{Chi}^{2}=11.36, \mathrm{df}=14(\mathrm{P}=0.66) ; \mathrm{I}^{2}=0 \%$} & $\begin{array}{r}0.2 \\
F\end{array}$ & $\begin{array}{c}0.5 \\
\text { Favours LG }\end{array}$ & $\begin{array}{l}1 \\
1 \\
\text { Favours OG }\end{array}$ \\
\hline
\end{tabular}

with strictly limiting inclusion and exclusion criteria to evaluate the safety and efficacy of LG in patients with AGC to determine whether LG is an acceptable alternative to OG. The quality scores of the included observational studies got 7 or more stars according to the NOS. These studies were primarily derived from the countries with the most widespread use of LG and mainly published in the past 5 years (2009-2013). Meta-analysis conducted based on this principle will contribute a more comprehensive and objective evaluation for the current status of LG treating AGC.

Reduction in the intraoperative blood loss is a consistent finding in studies comparing laparoscopic and open techniques in many different clinical situations. This is because laparoscopic surgery is more delicate than open surgery in providing perfect amplification. Regarding the operating time, LG is more time-consuming than OG. LG combined with lymphadenectomy is a complex operation and needs extensive technical expertise. Studies designed to estimate the learning curve have shown a significant reduction in operating time after about 50 LG cases [46-48]. Research from some large specialized centers reported that the operating time of LG was not longer than $O G$ in experienced hands [40,49]. Various modified techniques could help to simplify the procedure of reconstruction and shorten the operating time $[49,50]$. Therefore, researchers expect that with proficiency in

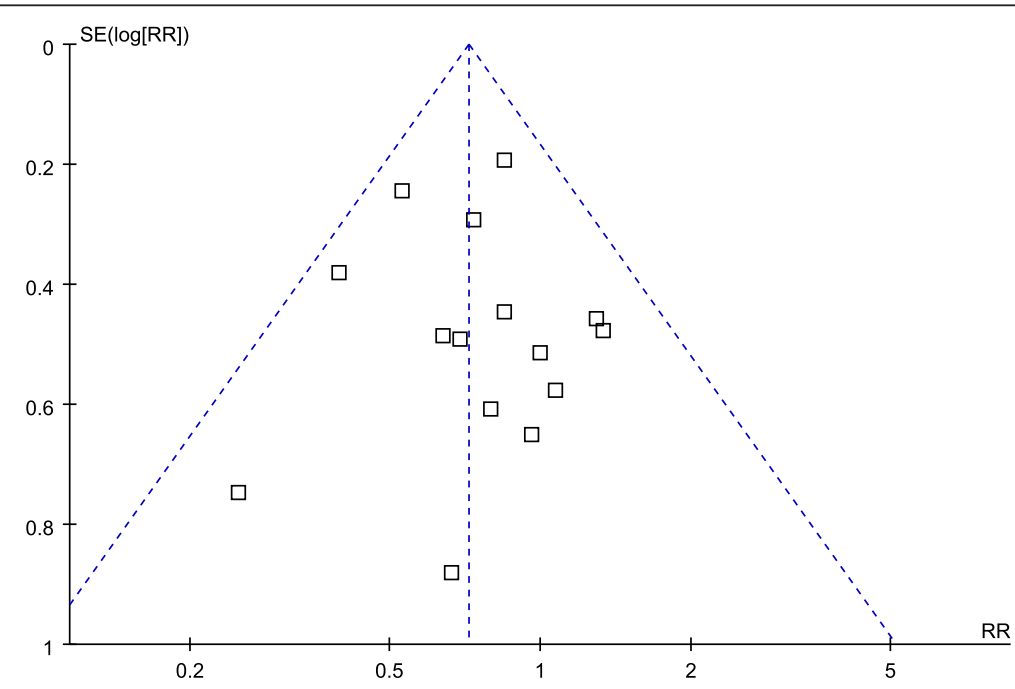

Figure 4 Funnel plot of the overall postoperative complications. 


\begin{tabular}{|c|c|c|c|c|c|c|c|}
\hline Study or Subgroup & $\begin{array}{r}\text { LG } \\
\text { Events }\end{array}$ & Total & $\begin{array}{r}\text { OG } \\
\text { Events }\end{array}$ & Total & Weight & $\begin{array}{l}\text { Risk Ratio } \\
\text { M-H, Random, } 95 \% \text { Cl Year }\end{array}$ & $\begin{array}{c}\text { Risk Ratio } \\
\text { M-H, Random, } 95 \% \text { CI }\end{array}$ \\
\hline Huscher & 11 & 30 & 10 & 29 & $3.7 \%$ & $1.06[0.53,2.11] 2005$ & \\
\hline Hur & 8 & 26 & 6 & 25 & $2.1 \%$ & $1.28[0.52,3.17] 2008$ & \\
\hline Du XH & 22 & 78 & 31 & 90 & $8.4 \%$ & $0.82[0.52,1.29] 2009$ & \\
\hline Hwang & 6 & 45 & 17 & 83 & $2.4 \%$ & $0.65[0.28,1.53] 2009$ & \\
\hline Du J & 19 & 82 & 23 & 94 & $6.2 \%$ & $0.95[0.56,1.61] 2010$ & \\
\hline Zhao & 147 & 346 & 141 & 313 & $58.0 \%$ & $0.94[0.79,1.12] 2011$ & \\
\hline Moisan & 5 & 31 & 4 & 31 & $1.2 \%$ & $1.25[0.37,4.22] 2012$ & \\
\hline Kim & 13 & 88 & 15 & 88 & $3.7 \%$ & $0.87[0.44,1.71] 2012$ & \\
\hline Chun & 4 & 52 & 7 & 67 & $1.3 \%$ & $0.74[0.23,2.38] 2012$ & \\
\hline Shinohara & 53 & 186 & 34 & 123 & $13.0 \%$ & $1.03[0.72,1.49] 2013$ & \\
\hline Total $(95 \% \mathrm{Cl})$ & & 964 & & 943 & $100.0 \%$ & $0.94[0.83,1.08]$ & \\
\hline Total events & 288 & & 288 & & & & \\
\hline \multicolumn{6}{|c|}{$\begin{array}{l}\text { Heterogeneity: } \mathrm{Tau}^{2}=0.00 ; \mathrm{Chi}^{2}=2.32, \mathrm{df}=9(\mathrm{P}=0.99) ; \mathrm{I}^{2}=0 \% \\
\text { Test for overall effect: } \mathrm{Z}=0.88(P=0.38)\end{array}$} & & $\begin{array}{ccc}0.50 .7 & 1 & 1.52 \\
\text { Favours LG } & \text { Favours OG }\end{array}$ \\
\hline
\end{tabular}

the laparoscopic technique and continuous improvement of equipment the time required for LG will become shorter [17].

One of the most striking findings was a reduced number of complications including surgical and medical ones in the LG versus OG group. Meta-analysis of the specified complications demonstrated that wound infections and ileus were significantly less common in the LG group. The reduced surface area of incisions and the manual handling of organs limit the risk of surgical site infections and ileus. It was not surprising that other surgical complications were not reduced because the laparoscopic technique, although less invasive, results in the same organ and lymphatic resection as the open procedure. Besides, the decreased medical complications could be explained by the reduced invasiveness of the laparoscopic technique and less pain after surgery. We also found that respiratory complications occurred in LG less often than in OG, although the difference was not significant $(P=0.09)$. The pain caused by a large incision as well as the use of tension sutures and abdominal bandages after laparotomy can make it difficult for patients to cough, expectorate and perform breathing exercises effectively, thus leading to such complications as pulmonary infection [51]. Reduced use of analgesic drugs, shortened time of abdominal cavity exposure and earlier postoperative activities are considered to be the main reasons for earlier gastrointestinal recovery from LG.

The concern about the technical difficulty of lymphadenectomy for perigastric lymph nodes is one of the major obstacles to accepting LG for AGC. Indeed, the adequacy of the radical resection should be evaluated by the extent of lymph node dissection performed and the number of retrieved lymph nodes. Our meta-analysis -revealed that there was no evident difference in the number of lymph nodes dissected between two groups, which was different from the results of some early metaanalyses $[16,17,52]$. In recent years, with improved equipment and increased surgeon experience, the number of lymph nodes dissected by LG has gradually increased $[8,53]$. Moreover, some researchers have reported not only a similar number of overall retrieved lymph nodes

Table 3 Systematic review of recurrence patterns and specific recurrent sites

\begin{tabular}{|c|c|c|c|c|c|c|c|c|c|}
\hline \multirow[t]{2}{*}{ Author } & \multirow[t]{2}{*}{ Group } & \multirow[t]{2}{*}{ Sample size } & \multicolumn{7}{|c|}{ Tumor recurrence } \\
\hline & & & Total & LR & LN & Liver & Lung & Bone & Peritoneum \\
\hline \multirow[t]{2}{*}{ Hur } & LG & 26 & 8 & 3 & 1 & 1 & & 2 & 1 \\
\hline & OG & 25 & 6 & & 5 & 1 & & & \\
\hline \multirow[t]{2}{*}{ Du J } & $\mathrm{LG}$ & 82 & 19 & 10 & & 5 & 4 & & \\
\hline & OG & 94 & 23 & 10 & & 7 & 4 & 2 & \\
\hline \multirow[t]{2}{*}{ Chun* } & $L G$ & 52 & 4 & & 3 & 2 & & 1 & \\
\hline & OG & 67 & 7 & 1 & 5 & 1 & & 1 & 3 \\
\hline \multirow[t]{2}{*}{$\mathrm{Kim}^{*}$} & LG & 88 & 13 & 2 & 4 & 6 & & 3 & 5 \\
\hline & OG & 88 & 15 & 2 & 4 & 4 & 3 & & 2 \\
\hline
\end{tabular}




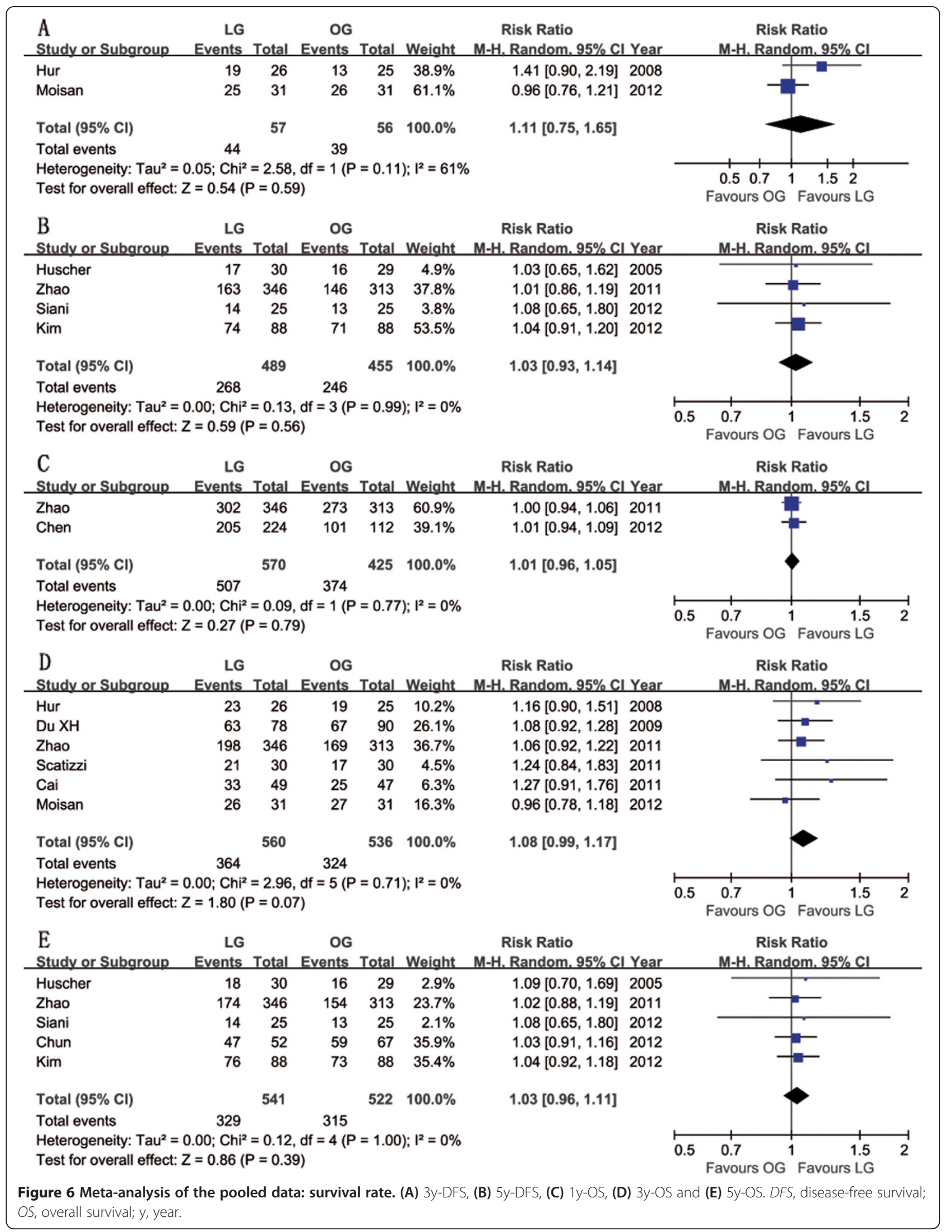


Table 4 Systematic review of long-term survival outcomes

\begin{tabular}{|c|c|c|c|c|c|}
\hline Author & Group & Follow-up (months) & Recurrence & DFS rate & OS rate \\
\hline \multirow[t]{2}{*}{ Huscher } & LG & $60(2-88)$ & 11 & 5-y: 57.3 & $5-y: 58.9$ \\
\hline & OG & $55(7-90)$ & 10 & 5-y: 54.8 & $5-y: 55.7$ \\
\hline \multirow[t]{2}{*}{ Hur } & LG & $29(6-47)$ & 8 & 3-y: 71.4 & $3-y: 88.2$ \\
\hline & OG & & 6 & 3-y: 53.4 & 3-y: 77.2 \\
\hline \multirow[t]{2}{*}{ Du XH } & LG & $25.2(4-58)$ & 22 & NR & $3-y: 81.2$ \\
\hline & OG & & 31 & NR & $3-y: 74.7$ \\
\hline \multirow[t]{2}{*}{ Hwang } & LG & $23(9-40)$ & 6 & NR & NR \\
\hline & OG & $23.5(8-41)$ & 17 & NR & NR \\
\hline \multirow[t]{2}{*}{ Du J } & LAG & $25(2-44)$ & 19 & NR & NR \\
\hline & OG & & 23 & NR & NR \\
\hline \multirow[t]{2}{*}{ Cai } & LG & $22.1(4-36)$ & NR & NR & $3-y: 67.1$ \\
\hline & OG & & NR & NR & 3-y: 53.8 \\
\hline \multirow[t]{2}{*}{ Scatizzi } & LG & $18(2-37)$ & NR & NR & $3-y: 70.9$ \\
\hline & OG & $18(7-42)$ & NR & NR & $3-y: 56.8$ \\
\hline \multirow[t]{2}{*}{ Shuang } & LG & $36.5(23-50)$ & NR & NR & NR \\
\hline & OG & $38.5(27-50)$ & NR & NR & NR \\
\hline \multirow[t]{2}{*}{ Zhao } & LG & $37(6-72)$ & 147 & $5-y: 47$ & $1-y: 87.2,3-y: 57.2,5-y: 50.3$ \\
\hline & OG & & 141 & 5-y: 46.8 & $1-y: 87.1,3-y: 54.1,5-y: 49.2$ \\
\hline \multirow[t]{2}{*}{ Chen } & LG & $19(1-48)$ & NR & NR & $1-y: 91.5$ \\
\hline & OG & & NR & NR & 1-y: 89.8 \\
\hline \multirow[t]{2}{*}{ Chun } & LG & $53.2(1-82.2)$ & 4 & NR & 5-y: 91.3 \\
\hline & OG & $60.4(7-91.7)$ & 7 & NR & $5-y: 88.6$ \\
\hline \multirow[t]{2}{*}{ Kim } & LG & $53.7(8.3-138.1)$ & 13 & 5-y: 84.6 & $5-y: 85.9$ \\
\hline & OG & $58.1(0.3-106.2)$ & 15 & 5-y: 81.1 & 5-y: 83.1 \\
\hline \multirow[t]{2}{*}{ Moisan } & LG & 28 & 5 & 3-y: 79.4 & $3-y: 82.3$ \\
\hline & OG & 40 & 4 & 3-y: 83.4 & $3-y: 86.9$ \\
\hline \multirow[t]{2}{*}{ Siani } & LG & 32.6 & NR & 5-y: 54.2 & 5-y: 55.7 \\
\hline & OG & 31.9 & NR & 5-y: 52.1 & $5-y: 52.9$ \\
\hline \multirow[t]{2}{*}{ Shinohara } & LG & $48.8(25-58.5)^{a}$ & 53 & $5-y: 65.8^{b}$ & $5-y: 68.1^{b}$ \\
\hline & OG & & 34 & $5-y: 62.0^{b}$ & $5-y: 63.7^{b}$ \\
\hline
\end{tabular}

Abbreviations: DFS Disease-free survival, OS Overall survival, $y$ Year, NR Not reported.

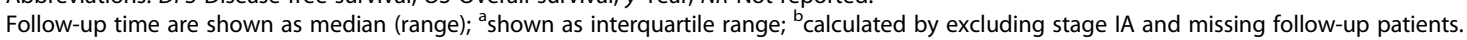

between LG and OG, but also a similar number of specific lymph nodes, such as group 7, 8a, 9, 11p, 12a and $14 \mathrm{v}$, which used to be considered difficult for laparoscopic dissection [54]. Park et al. [55] evaluated the longterm outcomes of 239 patients who underwent LG for the treatment of advanced gastric cancer. They found that the major recurrence was distant metastasis, whereas relapsed lymph nodes were most frequent in para-aortic or distant lymph node metastasis. Therefore, we believe that the dissection of lymph nodes around the stomach can be performed efficiently under laparoscope. Besides, splenic hilar lymph node dissection is one of the difficulties in upper and middle gastric cancer because the splenic vessels run circuitously, the branches vary substantially, and they are in a narrow and deep space. Therefore, it is easy to cause hemorrhage or spleen ischemia and further necrosis accidentally. Compared to laparotomy, laparoscopy allows the operator to complete the spleen hilum lymph node dissection under a clear field of view and helps to improve safety [56].

Cancer recurrence and the long-term survival rate are two critical outcomes for evaluating surgical interventions in oncological therapy. Based on the available data, postoperative cancer recurrence and the long-term survival rate in LG were similar to those in OG. Regarding the recurrence pattern, Song et al. [57] stated that the hematogenous pattern was most common after LG, followed by the locoregional pattern. This is consistent 
with the results of some of the included studies and other research [30]. The concern about dissemination of gastric cancer due to insufflated gas from pneumoperitoneum and port site or wound metastasis, although quite rare, has been emphasized. Port-site recurrence was seen in two of included studies [34,39]; however, it was not an event unique to LG, because there were also two cases of wound metastasis in OG group [39]. Zhao et al. and others $[39,58]$ stated that laparoscopic surgery does not promote abdominal or trocar implantation of gastric cancer. As previously mentioned, researchers indicated that LG did not increase the risk of perigastric lymph node recurrence compared to OG [55]. Sato et al. [59] analyzed the difference between OG and LG in relation to $D_{1}, D_{1+}$ or $D_{2}$ lymph node dissection using a hierarchical approach and found that the longterm results of LG were comparable to those of OG. Park et al. [55] analyzed the follow-up results of 239 cases of AGC treated with LG. The 5-year survival rates of $\mathrm{T}_{2}, \mathrm{~T}_{3}$ and $\mathrm{T}_{4}$ stage patients were $86.6 \%, 77.4 \%$ and $58.7 \%$, respectively, which is similar to that for concurrent laparotomy [60,61].

However, there were several limitations that must be taken into account when considering the above-men tioned results: (1) tumor depth and nodal status were risk factors for recurrence, and survival for patients with $\mathrm{pT}_{2}$ cancer has been reported to be better than that for patients with other advanced stage $[62,63]$. Two of the included studies were limited to $\mathrm{pT}_{2}$ stage patients $[32,41]$, and some of others mainly referred to stage IB-II or $\mathrm{pT}_{2-3}$ tumor invasion $[34,36,38,40,42,43,45]$. Hence, there should be an attitude of caution concerning laparoscopic resection of more advanced cases because relevant studies and clinical evidence are still deficient; (2) postoperative adjuvant chemotherapy has demonstrated a clear survival benefit compared to treatment with surgery alone $[3,4]$. However, some included studies failed to provide such information $[31,33,37,38,40-42,44]$, which might have affected the results; (3) the homogeneity test for the continuous variables exhibited substantial heterogeneity due to the inherent flaws of a retrospective study, the uneven surgical skills of the different surgeons as well as regional differences, etc.

\section{Conclusions}

The existing research shows that LG for AGC is safe and feasible, characterized by such advantages as less pain, fewer postoperative complications and rapid recovery. Moreover, our results suggest that the application of LG to this group results in adequate lymphadenectomy and similar recurrence and survival rates as OG. However, there were several limitations in this research. Therefore, the results mentioned above should be subject to verification by strictly designed, large-sample, multicenter RCTs with extended follow-up outcomes.

\section{Abbreviations}

LG: Laparoscopic gastrectomy; AGC: Advanced gastric cancer; RR: Risk ratio; WMD: Weighted mean differences; Cl: Confidence intervals; OG: Open gastrectomy; EGC: Early gastric cancer; RCT: Randomized controlled trial; NOS: Newcastle-Ottawa quality assessment scale; SD: Standard deviation; DFS: Disease-free survival; OS: Overall survival.

\section{Competing interests}

The authors declare that they have no competing interests.

\section{Authors' contributions}

CK and MYP designed the study; XXW and PY performed the research and retrieved data; ZYC, ZRC and WD collected the data; CK wrote the article; MYP proofread and revised the manuscript. All authors read and approved the final manuscript.

\section{Acknowledgements}

This work was supported by the key project grant from the Science and Technology Department of Zhejiang Province, China, no. 2011C13036-2.

Received: 10 June 2013 Accepted: 27 July 2013

Published: 8 August 2013

\section{References}

1. Yako-Suketomo H, Katanoda $\mathrm{K}$ : Comparison of time trends in stomach cancer mortality (1990-2006) in the world, from the WHO mortality database. Jpn J Clin Oncol 2009, 39:622-623.

2. Bertuccio P, Chatenoud L, Levi F, Praud D, Ferlay J, Negri E, Malvezzi M, La Vecchia C: Recent patterns in gastric cancer: a global overview. Int J Cancer 2009, 125:666-673.

3. Sakuramoto S, Sasako M, Yamaguchi T, Kinoshita T, Fujii M, Nashimoto A, Furukawa H, Nakajima T, Ohashi Y, Imamura H, Higashino M, Yamamura Y, Kurita A, Arai K, ACTS-GC Group: Adjuvant chemotherapy for gastric cancer with S-1, an oral fluoropyrimidine. N Engl J Med 2007, 357:1810-1820.

4. Bang YJ, Kim YW, Yang HK, Chung HC, Park YK, Lee KH, Lee KW, Kim YH, Noh SI, Cho JY, Mok YJ, Kim YH, Ji J, Yeh TS, Button P, Sirzén F, Noh SH, CLASSIC trial investigators: Adjuvant capecitabine and oxaliplatin for gastric cancer after $D_{2}$ gastrectomy (CLASSIC): a phase 3 open-label, randomised controlled trial. Lancet 2012, 379:315-321.

5. Catalano V, Labianca R, Beretta GD, Gatta G, de Braud F, van Cutsem E: Gastric cancer. Crit Rev Oncol Hematol 2009, 71:127-164.

6. Songun I, Putter $H$, Kranenbarg EM, Sasako M, van de Velde CJ: Surgical treatment of gastric cancer: 15-year follow-up results of the randomised nationwide Dutch D1D2 trial. Lancet Oncol 2010, 11:439-449.

7. Kitano S, Iso Y, Moriyama M, Sugimachi K: Laparoscopy-assisted Billroth I gastrectomy. Surg Laparosc Endosc 1994, 4:146-148.

8. Lee $\mathrm{JH}$, Han HS, Lee $\mathrm{JH}$ : A prospective randomized study comparing open vs laparoscopy-assisted distal gastrectomy in early gastric cancer: early results. Surg Endosc 2005, 19:168-173.

9. Kim YW, Baik YH, Yun YH, Nam BH, Kim DH, Choi IJ, Bae JM: Improved quality of life outcomes after laparoscopy-assisted distal gastrectomy for early gastric cancer: results of a prospective randomized clinical trial. Ann Surg 2008, 248:721-727.

10. Kim HH, Hyung WJ, Cho GS, Kim MC, Han SU, Kim W, Ryu SW, Lee HJ, Song KY: Morbidity and mortality of laparoscopic gastrectomy versus open gastrectomy for gastric cancer: an interim report-a phase III multicenter, prospective, randomized Trial (KLASS Trial). Ann Surg 2010, 251:417-420.

11. Eom BW, Kim YW, Lee SE, Ryu KW, Lee JH, Yoon HM, Cho SJ, Kook MC, Kim SJ: Survival and surgical outcomes after laparoscopy-assisted total gastrectomy for gastric cancer: case-control study. Surg Endosc 2012, 26:3273-3281.

12. Lee JH, Park do J, Kim HH, Lee HJ, Yang HK: Comparison of complications after laparoscopy-assisted distal gastrectomy and open distal gastrectomy for gastric cancer using the Clavien-Dindo classification. Surg Endosc 2012, 26:1287-1295.

13. Yasunaga $H$, Horiguchi $H$, Kuwabara K, Matsuda S, Fushimi K, Hashimoto $H$ Ayanian JZ: Outcomes after laparoscopic or open distal gastrectomy for early-stage gastric cancer: a propensity-matched analysis. Ann Surg 2013, 257:640-646. 
14. Miura S, Kodera Y, Fujiwara M, Ito S, Mochizuki Y, Yamamura Y, Hibi K, Ito K, Akiyama S, Nakao A: Laparoscopy-assisted distal gastrectomy with systemic lymph node dissection: a critical reappraisal from the viewpoint of lymph node retrieval. J Am Coll Surg 2004, 198:933-938.

15. Yakoub D, Athanasiou T, Tekkis P, Hanna GB: Laparoscopic assisted distal gastrectomy for early gastric cancer: is it an alternative to the open approach? Surg Oncol 2009, 18:322-333.

16. Chen XZ, Hu JK, Yang K, Wang L, Lu QC: Short-term evaluation of laparoscopy-assisted distal gastrectomy for predictive early gastric cancer: a meta-analysis of randomized controlled trials. Surg Laparosc Endosc Percutan Tech 2009, 19:277-284.

17. Ohtani H, Tamamori Y, Noguchi K, Azuma T, Fujimoto S, Oba H, Aoki T, Minami M, Hirakawa K: A meta-analysis of randomized controlled trials that compared laparoscopy-assisted and open distal gastrectomy for early gastric cancer. J Gastrointest Surg 2010, 14:958-964.

18. Liang Y, Li G, Chen P, Yu J, Zhang C: Laparoscopic versus open gastrectomy for early distal gastric cancer: a meta-analysis. ANZ J Surg 2011, 81:673-680.

19. Zeng YK, Yang ZL, Peng JS, Lin HS, Cai L: Laparoscopy-assisted versus open distal gastrectomy for early gastric cancer: evidence from randomized and nonrandomized clinical trials. Ann Surg 2012, 256:39-52.

20. Lee SE, Lee JH, Kook MC, Yoo JS, Ryu KW, Lee JS, Lee JY, Kim CG, Choi IJ, Park SR, Kim YW: $T_{2}$ or greater disease but were diagnosed preoperatively with early gastric cancer. Hepatogastroenterology 2008, 55:2282-2286

21. Grobmyer SR, Pieracci FM, Allen PJ, Brennan MF, Jaques DP: Defining morbidity after pancreaticoduodenectomy: use of a prospective complication grading system. J Am Coll Surg 2007, 204:356-364.

22. Stroup DF, Berlin JA, Morton SC, Olkin I, Williamson GD, Rennie D, Moher D, Becker BJ, Sipe TA, Thacker SB, Meta-Analysis Of Observational Studies in Epidemiology (MOOSE) Group: Meta-analysis of observational studies in epidemiology: a proposal for reporting. JAMA 2000, 283:2008-2012.

23. Clarke $M$, Horton R: Bringing it all together: Lancet-Cochrane collaborate on systematic reviews. Lancet 2001, 357:1728.

24. Hozo SP, Djulbegovic B, Hozo l: Estimating the mean and variance from the median, range, and the size of a sample. BMC Med Res Methodol 2005, 5:13.

25. Yoshimura F, Inaba K, Kawamura Y, Ishida Y, Taniguchi K, Isogaki J, Satoh S, Kanaya S, Sakurai Y, Uyama I: Clinical outcome and clinicopathological characteristics of recurrence after laparoscopic gastrectomy for advanced gastric cancer. Digestion 2011, 83:184-190.

26. Lin JX, Huang CM, Zheng CH, Li P, Xie JW, Wang JB, Lu J: Laparoscopy-assisted gastrectomy with $D_{2}$ lymph node dissection for advanced gastric cancer without serosa invasion: a matched cohort study from South China. World J Surg Oncol 2013, 11:4.

27. Hamabe A, Omori T, Tanaka K, Nishida T: Comparison of long-term results between laparoscopy-assisted gastrectomy and open gastrectomy with $\mathrm{D}_{2}$ lymph node dissection for advanced gastric cancer. Surg Endosc 2012, 26:1702-1709.

28. Gordon AC, Kojima K, Inokuchi M, Kato K, Sugihara K: Long-term comparison of laparoscopy-assisted distal gastrectomy and open distal gastrectomy in advanced gastric cancer. Surg Endosc 2013, 27:462-470.

29. Huang JL, Wei HB, Zheng ZH, Wei B, Chen TF, Huang Y, Guo WP, Hu B: Laparoscopy-assisted $D_{2}$ radical distal gastrectomy for advanced gastric cancer. Dig Surg 2010, 27:291-296.

30. MacLellan SJ, MacKay HJ, Ringash J, Jacks L, Kassam Z, Conrad T, Khalili I, Okrainec A: Laparoscopic gastrectomy for patients with advanced gastric cancer produces oncologic outcomes similar to those for open resection. Surg Endosc 2012, 26:1813-1821.

31. Huscher CG, Mingoli A, Sgarzini G, Sansonetti A, Di Paola M, Recher A, Ponzano C: Laparoscopic versus open subtotal gastrectomy for distal gastric cancer: five-year results of a randomized prospective trial. Ann Surg 2005, 241:232-237.

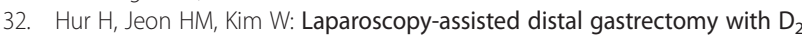
lymphadenectomy for $\mathrm{T}_{2 \mathrm{~b}}$ advanced gastric cancers: three years' experience. J Surg Oncol 2008, 98:515-519.

33. Du XH, Li R, Chen L, Shen D, Li SY, Guo Q: Laparoscopy-assisted $D_{2}$ radical distal gastrectomy for advanced gastric cancer: initial experience. Chin Med J (Engl) 2009, 122:1404-1407.

34. Hwang Sl, Kim HO, Yoo CH, Shin JH, Son BH: Laparoscopic-assisted distal gastrectomy versus open distal gastrectomy for advanced gastric cancer. Surg Endosc 2009, 23:1252-1258.
35. Du J, Zheng J, Li Y, Li J, Ji G, Dong G, Yang Z, Wang W, Gao Z: Laparoscopy-assisted total gastrectomy with extended lymph node resection for advanced gastric cancer-reports of 82 cases. Hepatogastroenterology 2010, 57:1589-1594.

36. Cai J, Wei D, Gao CF, Zhang CS, Zhang H, Zhao T: A prospective randomized study comparing open versus laparoscopy-assisted $D_{2}$ radical gastrectomy in advanced gastric cancer. Dig Surg 2011, 28:331-337.

37. Scatizzi M, Kroning KC, Lenzi E, Moraldi L, Cantafio S, Feroci F: Laparoscopic versus open distal gastrectomy for locally advanced gastric cancer: a case-control study. Updates Surg 2011, 63:17-23.

38. Shuang J, Qi S, Zheng J, Zhao Q, Li J, Kang Z, Hua J, Du J: A case-control study of laparoscopy-assisted and open distal gastrectomy for advanced gastric cancer. J Gastrointest Surg 2011, 15(1):57-62.

39. Zhao Y, Yu P, Hao Y, Qian F, Tang B, Shi Y, Luo H, Zhang Y: Comparison of outcomes for laparoscopically assisted and open radical distal gastrectomy with lymphadenectomy for advanced gastric cancer. Surg Endosc 2011, 25:2960-2966.

40. Chen QY, Huang CM, Lin JX, Zheng CH, Li P, Xie JW, Wang JB, Lu J: Laparoscopy-assisted versus open $D_{2}$ radical gastrectomy for advanced gastric cancer without serosal invasion: a case control study. World J Surg Oncol 2012, 10:248.

41. Chun HT, Kim KH, Kim MC, Jung GJ: Comparative study of laparoscopy-assisted versus open subtotal gastrectomy for $\mathrm{pT}_{2}$ gastric cancer. Yonsei Med J 2012, 53:952-959.

42. Kim KH, Kim MC, Jung GJ, Choi HJ, Jang JS, Kwon HC: Comparative analysis of five-year survival results of laparoscopy-assisted gastrectomy versus open gastrectomy for advanced gastric cancer: a case-control study using a propensity score method. Dig Surg 2012, 29:165-171.

43. Moisan F, Norero E, Slako M, Varas J, Palominos G, Crovari F, Ibanez L, Pérez G, Pimentel F, Guzmán S, Jarufe N, Boza C, Escalona A, Funke R: Completely laparoscopic versus open gastrectomy for early and advanced gastric cancer: a matched cohort study. Surg Endosc 2012, 26:661-672.

44. Siani LM, Ferranti F, De Carlo A, Quintiliani A: Completely laparoscopic versus open total gastrectomy in stage I-III/C gastric cancer: safety, efficacy and five-year oncologic outcome. Minerva Chir 2012, 67:319-326.

45. Shinohara T, Satoh S, Kanaya S, Ishida Y, Taniguchi K, Isogaki J, Inaba K, Yanaga $K$, Uyama I: Laparoscopic versus open $D_{2}$ gastrectomy for advanced gastric cancer: a retrospective cohort study. Surg Endosc 2013, 27:286-294.

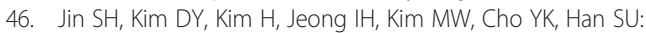
Multidimensional learning curve in laparoscopy-assisted gastrectomy for early gastric cancer. Surg Endosc 2007, 21:28-33.

47. Kunisaki C, Makino H, Yamamoto N, Sato T, Oshima T, Nagano Y, Fujii S, Akiyama H, Otsuka Y, Ono HA, Kosaka T, Takagawa R, Shimada H: Learning curve for laparoscopy-assisted distal gastrectomy with regional lymph node dissection for early gastric cancer. Surg Laparosc Endosc Percutan Tech 2008, 18:236-241.

48. Yoo CH, Kim HO, Hwang SI, Son BH, Shin JH, Kim H: Short-term outcomes of laparoscopic-assisted distal gastrectomy for gastric cancer during a surgeon's learning curve period. Surg Endosc 2009, 23:2250-2277.

49. Kim HS, Kim BS, Lee IS, Lee S, Yook JH, Kim BS: Comparison of totally laparoscopic total gastrectomy and open total gastrectomy for gastric cancer. J Laparoendosc Adv Surg Tech A 2013, 23:323-331.

50. Liao GQ, Ou XW, Liu SQ, Zhang SR, Huang W: Laparoscopy-assisted total gastrectomy with trans-orally inserted anvil (OrVil(TM)): A single institution experience. World J Gastroenterol 2013, 19:755-760.

51. Ephgrave KS, Kleiman-Wexler R, Pfaller M, Booth B, Werkmeister L, Young S Postoperative pneumonia: a prospective study of risk factors and morbidity. Surgery 1993, 114:815-821.

52. Kodera Y, Fujiwara M, Ohashi N, Nakayama G, Koike M, Morita S, Nakao A: Laparoscopic surgery for gastric cancer: a collective review with meta-analysis of randomized trials. J Am Coll Surg 2010, 211:677-686.

53. Lee JH, Yom CK, Han HS: Comparison of long-term outcomes of laparoscopy-assisted and open distal gastrectomy for early gastric cancer. Surg Endosc 2009, 23:1759-1763.

54. Song KY, Kim SN, Park CH: Laparoscopy-assisted distal gastrectomy with $D_{2}$ lymph node dissection for gastric cancer: technical and oncologic aspects. Surg Endosc 2008, 22:655-659.

55. Park Do J, Han SU, Hyung WJ, Kim MC, Kim W, Ryu SY, Ryu SW, Song KY, Lee HJ, Cho GS, Kim HH, Korean Laparoscopic Gastrointestinal Surgery Study (KLASS) Group: Long-term outcomes after laparoscopy-assisted 
gastrectomy for advanced gastric cancer: a large-scale multicenter retrospective study. Surg Endosc 2012, 26:1548-1553.

56. Wang JB, Huang CM, Zheng CH, Li P, Xie JW, Lin JX: Laparoscopic spleen-preserving No. 10 lymph node dissection for advanced proximal gastric cancer in left approach: a new operation procedure. World J Surg Oncol 2012, 10:241.

57. Song J, Lee HJ, Cho GS, Han SU, Kim MC, Ryu SW, Kim W, Song KY, Kim HH, Hyung WJ, Korean Laparoscopic Gastrointestinal Surgery Study (KLASS) Group: Recurrence following laparoscopy-assisted gastrectomy for gastric cancer: a multicenter retrospective analysis of 1,417 patients. Ann Surg Oncol 2010, 17:1777-1786.

58. Allieta R, Nardi M Jr, Brachet-Contul R, Millo P, Fabozzi M, Cornaglia S, Parini U: Laparoscopic gastrectomy for treatment of advanced gastric cancer: preliminary experience on 38 cases. Minerva Chir 2009, 64:445-456.

59. Sato H, Shimada M, Kurita N, Iwata T, Nishioka M, Morimoto S, Yoshikawa K, Miyatani T, Goto M, Kashihara H, Takasu C: Comparison of long-term prognosis of laparoscopy-assisted gastrectomy and conventional open gastrectomy with special reference to $D_{2}$ lymph node dissection. Surg Endosc 2012, 26:2240-2246.

60. Park CH, Song KY, Kim SN: Treatment results for gastric cancer surgery: 12 years' experience at a single institute in Korea. Eur I Surg Oncol 2008, 34:36-41.

61. Ahn HS, Lee HJ, Hahn S, Kim WH, Lee KU, Sano T, Edge SB, Yang HK: Evaluation of the seventh American Joint Committee on Cancer/ International Union Against Cancer Classification of gastric adenocarcinoma in comparison with the sixth classification. Cancer 2010, 116:5592-5598.

62. Sarela Al, Turnbull AD, Coit DG, Klimstra D, Brennan MF, Karpeh MS: Accurate lymph node staging is of greater prognostic importance than subclassification of the $T_{2}$ category for gastric adenocarcinoma. Ann Surg Oncol 2003, 10:783-791.

63. Otsuji E, Kuriu Y, Ichikawa D, Okamoto K, Hagiwara A, Yamagishi H: Characteristics of gastric carcinoma invading the muscularis propria. J Surg Oncol 2005, 92:104-108.

doi:10.1186/1477-7819-11-182

Cite this article as: Chen et al: Systematic review and meta-analysis of laparoscopic and open gastrectomy for advanced gastric cancer. World Journal of Surgical Oncology 2013 11:182.

\section{Submit your next manuscript to BioMed Central and take full advantage of:}

- Convenient online submission

- Thorough peer review

- No space constraints or color figure charges

- Immediate publication on acceptance

- Inclusion in PubMed, CAS, Scopus and Google Scholar

- Research which is freely available for redistribution 